\title{
Moment approach to the bootstrap current in nonaxisymmetric toroidal plasmas using $\delta f$ Monte Carlo methods
}

\author{
A. Matsuyama ${ }^{1, a)}$ M. Yu. Isaev ${ }^{2}$ K. Y. Watanabe, ${ }^{3}$ K. Hanatani, ${ }^{4}$ Y. Suzuki, ${ }^{3}$ N. Nakajima, ${ }^{3}$ \\ W. A. Cooper, ${ }^{5}$ and T. M. Tran ${ }^{5}$ \\ ${ }^{1}$ Graduate School of Energy Science, Kyoto University, Gokasho, Uji, Kyoto 611-0011, Japan \\ ${ }^{2}$ Nuclear Fusion Institute, RRC Kurchatov Institute, 123182 Moscow, Russia \\ ${ }^{3}$ National Institute for Fusion Science, Toki, Gifu 509-5292, Japan \\ ${ }^{4}$ Institute of Advanced Energy, Kyoto University, Gokasho, Uji, Kyoto 611-0011, Japan \\ ${ }^{5}$ Centre de Recherches en Physique des Plasmas, Association Euratom-Suisse, \\ Ecole Polytechnique Fédérale de Lausanne, CH1015 Lausanne, Switzerland
}

(Received 4 March 2009; accepted 27 March 2009; published online 7 May 2009)

To evaluate the bootstrap current in nonaxisymmetric toroidal plasmas quantitatively, a $\delta f$ Monte Carlo method is incorporated into the moment approach. From the drift-kinetic equation with the pitch-angle scattering collision operator, the bootstrap current and neoclassical conductivity coefficients are calculated. The neoclassical viscosity is evaluated from these two monoenergetic transport coefficients. Numerical results obtained by the $\delta f$ Monte Carlo method for a model heliotron are in reasonable agreement with asymptotic formulae and with the results obtained by the variational principle. (c) 2009 American Institute of Physics. [DOI: 10.1063/1.3121223]

\section{INTRODUCTION}

The bootstrap current in nonaxisymmetric toroidal devices has significant influence on the confinement properties of a plasma, such as equilibrium and stability, through the change in rotational transform profiles and magnetic field spectra. ${ }^{1-4}$ Because the bootstrap current is induced predominantly by the trapping and detrapping of particles in the lowcollisionality regime, 5,6 a collision operator of the driftkinetic equation can be modeled appropriately by the pitchangle scattering term. With this assumption, a bootstrap current coefficient $D_{31}$ has been calculated numerically for various nonaxisymmetric configurations. ${ }^{7}$ This coefficient $D_{31}$ is proportional to the geometric factor, ${ }^{8}$ which characterizes the relative amplitude and sign of the bootstrap current for a given toroidal configuration. For example, the $\delta f$ Monte Carlo method ${ }^{9,10}$ constitutes an efficient numerical technique to calculate $D_{31}$ by guiding-center particle simulations.

To evaluate the bootstrap current in a satisfactory manner, however, we also need to take into account the momentum conservation law of like-particle collisions and the coupling between electrons and ions. Consequently, the $\delta f$ Monte Carlo method for $D_{31}$, which relies only on the pitchangle scattering operator, is inadequate to evaluate the bootstrap current quantitatively. In the analytical theory by Shaing and Callen, ${ }^{8}$ the bootstrap current $\left\langle J_{\|} B\right\rangle$ on each flux surface is determined from the momentum and heat-flux balance equations along the field lines. To treat such macroscopic balances correctly, one must simulate ensembles of test electrons and ions with Maxwellian distributions and to use the like and unlike-particle collision operators that preserve the conservation laws. To date, such a self-consistent Monte Carlo simulation of the bootstrap current has been

${ }^{a)}$ Electronic mail: matuyama@center.iae.kyoto-u.ac.jp reported only for tokamaks ${ }^{11}$ but not for nonaxisymmetric devices.

Recently, Sugama and Nishimura ${ }^{12,13}$ developed another method to evaluate the bootstrap current, which overcomes the above difficulties. They showed that parallel viscosities $\left\langle\mathbf{B} \cdot\left(\nabla \cdot \boldsymbol{\pi}_{a}\right)\right\rangle$ and $\left\langle\mathbf{B} \cdot\left(\nabla \cdot \boldsymbol{\Theta}_{a}\right)\right\rangle$ can be evaluated numerically using the drift-kinetic equation solver (DKES) code ${ }^{14,15}$ substituting these viscosity terms into the moment equations, ${ }^{16}$ the bootstrap current is determined algebraically without breaking the conservation laws. While based on the pitchangle scattering approximation, the important feature of the DKES code is that all the elements of the monoenergetic transport matrix $D_{i j}(i, j=1,3)$ can be calculated by the variational principle. We emphasize that this moment approach takes computational advantages of the pitch-angle scattering operator and yet satisfies the physical requirement of the conservation laws of the linearized Fokker-Planck collision operator. ${ }^{12,17}$

In the moment approach by Sugama and Nishimura, not only the nondiagonal element $D_{31}$ but also the diagonal one $D_{33}$ of the monoenergetic transport matrix is required to calculate the neoclassical viscosity. To our knowledge, $D_{33}$, which corresponds to a (monoenergetic) neoclassical conductivity coefficient, has been calculated so far only by the DKES code. We mention that this diagonal term $D_{33}$ can be related to the damping rate of neoclassical flows along the field lines and is indispensable to determine the neoclassical viscosity. Considering the $\delta f$ Monte Carlo method for $D_{31}$, an extension of the method to the calculation of $D_{33}$ will be useful to evaluate the bootstrap current quantitatively with the moment approach.

The purpose of this paper is twofold: (i) to develop a $\delta f$ Monte Carlo method that calculates the monoenergetic transport coefficient $D_{33}$ and (ii) to incorporate this method into the moment approach for evaluating the bootstrap current $\left\langle J_{\|} B\right\rangle$. Following the moment approach for the DKES code, 
the neoclassical parallel viscosities $\left\langle\mathbf{B} \cdot\left(\nabla \cdot \boldsymbol{\pi}_{a}\right)\right\rangle$ and $\left\langle\mathbf{B} \cdot\left(\nabla \cdot \boldsymbol{\Theta}_{b}\right)\right\rangle$ are obtained from an output of the $\delta f$ Monte Carlo method, i.e., $D_{31}$ and $D_{33}$. We have implemented the $\delta f$ weighting scheme presented here into the VENUS $+\delta f$ code $^{9}$ developed at CRPP (Switzerland). The numerical calculation of the neoclassical viscosities is tested for a magnetic-field model of the large helical device (LHD). ${ }^{18}$

This paper is organized as follows. In Sec. II, the moment approach to evaluate the bootstrap current using the $\delta f$ Monte Carlo method is described. We begin with the driftkinetic equation with the pitch-angle scattering approximation and derive a $\delta f$ weighting scheme to calculate $D_{31}$ and $D_{33}$. The evaluation of the bootstrap current from these transport coefficients is also discussed. In Sec. III, we describe the numerical procedure and show benchmarking results with asymptotic formulae and with the DKES code. Finally, the conclusion will be given in Sec. IV. In the Appendix, an additional topic on the present $\delta f$ Monte Carlo method will be discussed.

\section{MOMENT APPROACH WITH A $\delta f$ MONTE CARLO METHOD}

\section{A. Basic formalism}

We first consider the drift-kinetic theory based on the pitch-angle scattering approximation. In this paper, we use the coordinate system $(\mathbf{x}, v, \xi)$, where $\mathbf{x}$ is the guiding-center position, $v$ is the particle velocity, and $\xi \equiv v_{\|} / v$ is the pitch variable. For transport applications, the guiding-center position is usually written in Boozer coordinates, ${ }^{19}(s, \theta, \zeta): s$ is the surface label, $\theta$ is the poloidal angle, and $\zeta$ is the toroidal angle. The Jacobian of the Boozer coordinate is denoted by $\mathcal{J}_{B}$. The flux surface average in this coordinate is defined by $\langle A\rangle \equiv\left(\oint \oint d \theta d \zeta \mathcal{J}_{B} A\right) /\left(\oint \oint d \theta d \zeta \mathcal{J}_{B}\right)$.

The neoclassical transport coefficients are calculated from a steady-state solution of the linearized drift-kinetic equation. Let $f=f_{0}+\delta f$, where

$$
f_{0} \equiv f_{M}=\frac{n(s)}{\pi^{3 / 2} v_{T}^{3}} \exp (-K)
$$

is a local Maxwellian with the density $n(s)$ and the temperature $T(s)$ and $\delta f$ is the perturbed part of a gyroaveraged distribution function; $v_{T}=(2 T / m)^{1 / 2}$ is the local thermal velocity and $K=m v^{2} / 2 T$ is the normalized kinetic energy. Approximating a collision operator by the pitch-angle scattering term, the linearized drift-kinetic equation is written in terms of $g \equiv \delta f / f_{0}$ as

$$
\left(V_{\|}-C\right) g=\mathbf{v}_{d} \cdot \nabla s A_{1}+B v \xi A_{E},
$$

where

$$
\begin{aligned}
& V_{\|}=v \xi \mathbf{b} \cdot \nabla-\frac{1}{2} v\left(1-\xi^{2}\right)(\mathbf{b} \cdot \nabla \ln B) \frac{\partial}{\partial \xi}, \\
& C=\frac{\nu_{D}}{2} \frac{\partial}{\partial \xi}\left(1-\xi^{2}\right) \frac{\partial}{\partial \xi},
\end{aligned}
$$

$$
\begin{aligned}
& A_{1}=-\frac{1}{n} \frac{\partial n}{\partial s}-\frac{e}{T} \frac{\partial \Phi}{\partial s}, \\
& A_{E}=\frac{e}{T} \frac{\left\langle B E_{\|}\right\rangle}{\left\langle B^{2}\right\rangle} .
\end{aligned}
$$

Here, $B \equiv|\mathbf{B}|$ is the equilibrium magnetic-field strength, $\mathbf{b} \equiv \mathbf{B} / B$ is the unit vector along the field line, $E_{\|}$is the parallel electric field, and $\Phi$ is the electrostatic potential. In Eq. (2), $\mathbf{v}_{d}$ denotes the guiding-center drift velocity, while in the left-hand side of Eq. (2) we explicitly assume the steadystate solution such that $\partial g / \partial t \equiv 0$. The pitch-angle scattering term $C$ is characterized by the deflection frequency $\nu_{D}$, and the operator $V_{\|}$only includes terms up to the zeroth order of $\rho_{p} / a \ll 1$, where $\rho_{p}=m v / e B_{p}$ is the poloidal gyroradius (with the characteristic poloidal magnetic field $B_{p}$ ) and $a$ is the plasma minor radius. Equation (2) is derived with the neoclassical ordering, i.e., $\rho_{p} / a \ll 1$, which means the radial orbit width is assumed to be small enough and the transport coefficients can be determined locally on a flux surface. The temperature gradient $\partial T / \partial s$ is also neglected here. The monoenergetic transport matrix $D_{i j}(K)(i, j=1,3)$, which is a function of the normalized kinetic energy $K$ or equivalently that of the collisionality $\nu_{D} / v$, is defined in the DKES code $^{14,15}$ as follows:

$$
D_{i j}(K) \equiv\left(\sigma_{i}^{+}, F_{j}^{+}\right)+\left(\sigma_{i}^{+}, F_{j}^{-}\right) \quad(i, j=1,3) .
$$

In the DKES code, the drift-kinetic equation [Eq. (2)] is separated in terms of the symmetric (+) and antisymmetric $(-)$ parts of the distribution function $F_{j}^{ \pm}(j=1,3)$ with respect to the time-reversal operation such that

$$
\begin{aligned}
& V_{\|} F_{j}^{-}-C F_{j}^{+}=\sigma_{j}^{+}, \\
& V_{\|} F_{j}^{+}-C F_{j}^{-}=0 \quad(j=1,3),
\end{aligned}
$$

with

$$
\begin{aligned}
& \sigma_{1}^{+}=-\mathbf{v}_{d} \cdot \nabla s=-\frac{2 v^{2}}{3 \Omega}\left[1+\frac{1}{2} P_{2}(\xi)\right] \mathbf{b} \times \nabla \ln B \cdot \nabla s, \\
& \sigma_{3}^{+}=V_{\|}\left(B v \xi / \nu_{D}\right)=\frac{v^{2}}{\nu_{D}} P_{2}(\xi) \mathbf{B} \cdot \nabla \ln B .
\end{aligned}
$$

Here, $\Omega=e B / m$ is the gyrofrequency and $P_{2}(\xi) \equiv\left(3 \xi^{2}\right.$ $-1) / 2$ denotes the second-order Legendre polynomial. The parentheses in Eq. (7) denote the inner-product operation defined by

$$
(\alpha, \beta)=\frac{1}{2} \int_{-1}^{1} d \xi\langle\alpha \beta\rangle,
$$

where $\langle\cdot\rangle$ denotes the flux surface average. Note that the time-reversal symmetric properties of the operators $V_{\|}$and $C$ with respect to this inner-product operation apply such that

$$
\left(V_{\|} \alpha, \beta\right)=-\left(\alpha, V_{\|} \beta\right), \quad(C \alpha, \beta)=(\alpha, C \beta) .
$$

Equation (8) is derived with the adjoint equation ${ }^{14,15}$ of the drift-kinetic equation, which prevents us from solving Eq. (8) by $\delta f$ Monte Carlo methods. Hence we need to con- 
struct an alternative set of the drift-kinetic equations that can be solved as an initial-value problem using guiding-center particle simulations. We now decompose the dependence of the perturbed distribution function $g$ into two independent thermodynamic forces $A_{1}$ and $A_{E}$ so that $g=g_{1} A_{1}+g_{E} A_{E}$, which yields

$$
\begin{aligned}
& \left(V_{\|}-C\right) g_{1}=-\sigma_{1}^{+}, \\
& \left(V_{\|}-C\right) g_{E}=B v \xi .
\end{aligned}
$$

Comparing Eqs. (13) and (14) with Eq. (8), we have obtained the following relations:

$$
\begin{aligned}
& g_{1}=-\left(F_{1}^{+}+F_{1}^{-}\right), \\
& g_{E}=-\left(F_{3}^{+}+F_{3}^{-}\right)+B v \xi / \nu_{D} .
\end{aligned}
$$

If we take the inner-product operation of $e B v \xi$ with the solutions of Eqs. (13) and (14), the current carried by test particles in a simulation, which is here denoted by $\hat{j} \equiv \hat{j}_{\text {bs }}+\hat{j}_{\mathrm{Oh}}$, is explicitly written in terms of $D_{31}$ and $D_{33}$,

$$
\begin{aligned}
& \hat{j}_{\mathrm{bs}} \equiv e\left(B v \xi, g_{1}\right) A_{1}=-e D_{31} A_{1}, \\
& \hat{j}_{\mathrm{Oh}} \equiv e\left(B v \xi, g_{E}\right) A_{E}=-e D_{33} A_{E}+\frac{e\left\langle B^{2}\right\rangle v^{2}}{3 \nu_{D}} A_{E},
\end{aligned}
$$

where $\hat{j}_{\text {bs }}$ and $\hat{j}_{\text {Oh }}$ denote the monoenergetic bootstrap and Ohmic currents, respectively. Equation (17) allows us to calculate the bootstrap current coefficient $D_{31}$ from the steadystate solution $g_{1}$. On the other hand, Eq. (18) yields the neoclassical conductivity coefficient $D_{33}$, but the inclusion of the second term in the right-hand side of Eq. (18) is unfavorable with respect to numerical accuracy. This term comes from the classical Spitzer distribution $g_{S} \equiv B v \xi / \nu_{D}$, where $g_{S}$ is the solution of the equation ${ }^{15}$ such that $-C g_{S}=B v \xi$. To eliminate this Spitzer current from Eq. (18), we introduce the driftkinetic equation such that

$$
\left(V_{\|}-C\right) g_{3}=-\sigma_{3}^{+},
$$

where

$$
g_{3} \equiv g_{E}-g_{S}=-\left(F_{3}^{+}+F_{3}^{-}\right) .
$$

Using the steady-state solution of Eq. (19), we obtain the monoenergetic neoclassical-conductive current $\hat{j}_{\text {nc }}$ as

$$
\hat{j}_{\mathrm{nc}} \equiv e\left(B v \xi, g_{3}\right) A_{E}=-e D_{33} A_{E} .
$$

Equation (21) is more appropriate than Eq. (18) to calculate $D_{33}$ because in the collisional regime, the classical Spitzer current $\hat{j}_{\mathrm{Sp}} \equiv e\left\langle B^{2}\right\rangle v^{2} A_{E} /\left(3 \nu_{D}\right)$ dominates over the first term in Eq. (18) in the expression for $\hat{j}_{\mathrm{Oh}}$. This prohibits the accurate evaluation of $D_{33}$ using Eq. (18). As a result, we find that the monoenergetic transport coefficients $D_{31}$ and $D_{33}$ are calculated from the steady-state solution of the drift-kinetic equations [Eqs. (13) and (19)]. It should be noted that these equations are consistent with the DKES code through the explicit relations in Eqs. (15) and (20).

\section{B. $\delta f$ weighting scheme}

Next, we develop a $\delta f$ weighting scheme to solve the drift-kinetic equations of Eqs. (13) and (19). It is well known that the introduction of marker weights into Monte Carlo simulations reduces the statistical noise up to the order of $\delta f / f$. Here, to solve Eqs. (13) and (19) simultaneously, we introduce two marker weights $w_{1} \equiv g_{1} / F_{m}$ and $w_{3} \equiv g_{3} / F_{m}$, where $F_{m}(\mathbf{x}, \xi)$ denotes a distribution function of test particles. We note that the particle velocity $v$ is now manifestly a constant of motion, i.e., $v=$ const. These two marker weights, $w_{1}$ and $w_{3}$, are assigned to test particles and are updated by the integration along the test-particle trajectories. The time evolution of $w_{i}$ is represented in terms of the Lagrangian derivative as

$$
\frac{D w_{i}}{D t}=-\sigma_{i}^{+} \quad(i=1,3) .
$$

For $i=1$, the right-hand side of Eq. (22) corresponds to the radial excursion of test particles such that $\sigma_{1}^{+}=-d s / d t$. The Lagrangian derivative $D / D t$ is given by

$$
\frac{D}{D t} \equiv \frac{\partial}{\partial t}+V_{\|}-C
$$

where the equilibrium trajectory defined by Eq. (23) can be simulated with the Monte Carlo method as will be described later. At each time step of the simulation, the monoenergetic transport coefficients $D_{31}$ and $D_{33}$ can be calculated by the $\delta f$ Monte Carlo integral. ${ }^{20}$ Using Eqs. (17) and (21), we obtain

$$
D_{31}=-\frac{\sum_{n=1}^{N} \mathcal{J}_{B}\left(\mathbf{x}_{n}\right) B\left(\mathbf{x}_{n}\right) v \xi_{n} w_{1}^{(n)}}{\sum_{n=1}^{N} \mathcal{J}_{B}\left(\mathbf{x}_{n}\right)}
$$

and

$$
D_{33}=-\frac{\sum_{n=1}^{N} \mathcal{J}_{B}\left(\mathbf{x}_{n}\right) B\left(\mathbf{x}_{n}\right) v \xi_{n} w_{3}^{(n)}}{\sum_{n=1}^{N} \mathcal{J}_{B}\left(\mathbf{x}_{n}\right)},
$$

where $N$ is the number of test particles. All the quantities in the right-hand sides in Eqs. (24) and (25) are given along the trajectory in $(\mathbf{x}, \xi)$ space. To derive Eqs. (24) and (25), we have used here that

$$
\begin{aligned}
g(\mathbf{x}, \xi)-g_{S}= & \frac{1}{C_{N}} \sum_{n=1}^{N}\left[w_{1}^{(n)} \delta\left(\mathbf{x}-\mathbf{x}_{n}\right) \delta\left(\xi-\xi_{n}\right) A_{1}\right. \\
& \left.+w_{3}^{(n)} \delta\left(\mathbf{x}-\mathbf{x}_{n}\right) \delta\left(\xi-\xi_{n}\right) A_{E}\right],
\end{aligned}
$$

where $C_{N} \equiv N / 2$ is a normalization constant to ensure $\frac{1}{2} \int d \mathbf{x} \int_{-1}^{1} d \xi F_{m}(\mathbf{x}, \xi)=1$ and $\delta$ is a Dirac delta function. The separation of the marker weight into the thermodynamic forces has been proposed by Tessarotto et al. ${ }^{21}$ for gyrokinetic theory. We have applied this method to the monoenergetic drift-kinetic equation in Eq. (2) such that $\left(g-g_{S} A_{E}\right) /$ $F_{m}=w_{1} A_{1}+w_{3} A_{3}$. The monoenergetic transport coefficients $D_{31}$ and $D_{33}$ are therefore obtained from Eqs. (24) and (25). The numerical implementation of the present $\delta f$ weighting scheme is discussed in Sec. III A.

The $\delta f$ weighting scheme (26) involves the standard $\delta f$ scheme to calculate the part corresponding to $D_{31}$. To see 
this, we consider the standard one-weight $\delta f$ scheme ${ }^{11,22,23}$ based on Eq. (2), in which a marker weight $w \equiv g / F_{m}$ is given by

$$
g(\mathbf{x}, \xi)=\frac{1}{C_{N}} \sum_{n=1}^{N} w^{(n)} \delta\left(\mathbf{x}-\mathbf{x}_{n}\right) \delta\left(\xi-\xi_{n}\right) .
$$

The inner-product operation of $e B v \xi$ with $g$ yields

$$
\hat{j} \equiv e(B v \xi, g)=-e D_{31} A_{1}-e D_{33} A_{E}+\frac{e\left\langle B^{2}\right\rangle v^{2}}{3 \nu_{D}} A_{E} .
$$

In the calculation of the bootstrap current coefficient $D_{31}$, the parallel thermodynamic force is normally neglected, i.e., $A_{E}=0$. Introducing the two marker weights $w_{1}$ and $w_{3}$, we can calculate $D_{31}$ and $D_{33}$ independently excluding a contribution from the Spitzer distribution for all collisionality regimes. When we apply the $\delta f$ Monte Carlo method to the moment approach like that done with the DKES code, the latter feature becomes important for accurate evaluation of $D_{33}$.

\section{Evaluation of the bootstrap current}

Using the $\delta f$ Monte Carlo method described above, the bootstrap current $\left\langle J_{\|} B\right\rangle$ can be evaluated with the moment approach. Here, we follow the formulation by Sugama and Nishimura. ${ }^{12}$ We consider a plasma that consists of electrons and a single species of ions. In Secs. II A and II B, we have not specified particle species because under the pitch-angle scattering approximation, the monoenergetic transport coefficients depend only on the collisionality and are irrelevant to the particle species in these calculations. With the moment approach, nonetheless, the transport coefficients obtained as a function of $\nu_{D} / v$ can be used to describe the neoclassical transport for multispecies plasmas. ${ }^{24}$

To determine the bootstrap current, we need to solve the surface-averaged momentum and heat-flux balance equations for electrons and ions,

$$
\begin{aligned}
& \left\langle\mathbf{B} \cdot\left(\nabla \cdot \boldsymbol{\pi}_{a}\right)\right\rangle-n_{a} e_{a}\left\langle B E_{\|}\right\rangle=\left\langle B F_{\| a 1}\right\rangle, \\
& \left\langle\mathbf{B} \cdot\left(\nabla \cdot \boldsymbol{\Theta}_{a}\right)\right\rangle=\left\langle B F_{\| a 2}\right\rangle .
\end{aligned}
$$

The neoclassical parallel viscosities $\left\langle\mathbf{B} \cdot\left(\nabla \cdot \boldsymbol{\pi}_{a}\right)\right\rangle$ and $\left\langle\mathbf{B} \cdot\left(\nabla \cdot \boldsymbol{\Theta}_{a}\right)\right\rangle$ in the left-hand side of Eqs. (29) and (30) are related to the plasma flows and the thermodynamic forces through the viscosity-flow relations

$$
\begin{aligned}
{\left[\begin{array}{c}
\left\langle\mathbf{B} \cdot\left(\nabla \cdot \boldsymbol{\pi}_{a}\right)\right\rangle \\
\left\langle\mathbf{B} \cdot\left(\nabla \cdot \boldsymbol{\Theta}_{a}\right)\right\rangle
\end{array}\right]=} & {\left[\begin{array}{ll}
M_{a 1} & M_{a 2} \\
M_{a 2} & M_{a 3}
\end{array}\right]\left[\begin{array}{c}
\left\langle u_{\| a} B\right\rangle /\left\langle B^{2}\right\rangle \\
\frac{2}{5 p_{a}}\left\langle q_{\| a} B\right\rangle\left\langle\left\langle B^{2}\right\rangle\right.
\end{array}\right] } \\
& +\left[\begin{array}{ll}
N_{a 1} & N_{a 2} \\
N_{a 2} & N_{a 3}
\end{array}\right]\left[\begin{array}{c}
X_{a 1} \\
X_{a 2}
\end{array}\right]
\end{aligned}
$$

where

$$
X_{a 1} \equiv-\frac{1}{n_{a}} \frac{\partial p_{a}}{\partial s}-e_{a} \frac{\partial \Phi}{\partial s},
$$

$$
X_{a 2} \equiv-\frac{\partial T_{a}}{\partial s} .
$$

Here, $\left\langle u_{\| a} B\right\rangle$ and $\left\langle q_{\| a} B\right\rangle$ are the surface-averaged flows of particles and heat, and $p_{a}=n_{a} T_{a}$ is the plasma pressure. The parallel friction forces $\left\langle B F_{\| a 1}\right\rangle$ and $\left\langle B F_{\| a 2}\right\rangle$ in the right-hand side of Eqs. (29) and (30) can also be expressed in terms of the coefficients $l_{j k}^{a b}$ by the friction-force relations ${ }^{16}$

$$
\left[\begin{array}{c}
\left\langle B F_{\| a 1}\right\rangle \\
\left\langle B F_{\| a 2}\right\rangle
\end{array}\right]=\sum_{b}\left[\begin{array}{cc}
l_{11}^{a b} & -l_{12}^{a b} \\
-l_{21}^{a b} & l_{22}^{a b}
\end{array}\right]\left[\begin{array}{c}
\left\langle B u_{\| b}\right\rangle \\
\frac{2}{5 p_{a}}\left\langle B q_{\| b}\right\rangle
\end{array}\right],
$$

which are valid in any toroidal geometry and in any collisionality regime. These friction-flow relations satisfy ${ }^{16,25}$ the self-adjointness $l_{j k}^{a b}=l_{k j}^{b a}$ and the momentum conservation laws $\Sigma_{a} a_{1 k}^{a b}=0$. If one substitutes the viscosity-flow [Eq. (31)] and the friction-flow relations [Eq. (34)] into the parallel momentum and heat-flux balance equations [Eqs. (29) and (30)], the surface-averaged parallel current $\left\langle J_{\|} B\right\rangle$ is obtained by

$$
\begin{aligned}
\frac{\left\langle J_{\|} B\right\rangle}{\left\langle B^{2}\right\rangle^{1 / 2}}= & L_{E 1}^{e} X_{e 1}+L_{E 2}^{e} X_{e 2}+L_{E 1}^{i} X_{i 1}+L_{E 2}^{i} X_{i 2} \\
& +\left(L_{E E}+\sigma_{S}\right) X_{E},
\end{aligned}
$$

where the linear transport coefficients $L_{E 1}^{a}$ and $L_{E 2}^{a}(a=e, i)$ are the (full energetic) electron and ion bootstrap current coefficients, $L_{E E}$ is the neoclassical conductivity, and $\sigma_{S}$ is the classical Spitzer conductivity. The parallel thermodynamic force $X_{E}$ is defined as

$$
X_{E} \equiv \frac{\left\langle B E_{\|}\right\rangle}{\left\langle B^{2}\right\rangle^{1 / 2}}
$$

Equation (35) determines the bootstrap current $\left\langle J_{\|} B\right\rangle$ locally on a flux surface for a given plasma profile in nonaxisymmetric devices.

Although the general expression of the friction-flow relations is obtained, the viscosity-flow relations must be specified by the solution of the drift-kinetic equation. Conventionally in nonaxisymmetric systems, the viscosity-flow relations have been obtained by the asymptotic expansion ${ }^{8,25}$ of the drift-kinetic equation. In the present moment approach, this step is replaced by the $\delta f$ Monte Carlo method. Following the moment approach by Sugama and Nishimura, we evaluate the two energy-dependent viscosity coefficients $M_{a}(K)$ and $N_{a}(K)$ in terms of the monoenergetic transport coefficients $D_{31}$ and $D_{33}$ as

$$
\begin{aligned}
M_{a}(K)= & \frac{m_{a}^{2}}{T_{a}}\left[\nu_{D}^{a}(K)\right]^{2} D_{33}(K) \\
& \times\left[1-\frac{3 m_{a} \nu_{D}^{a}(K) D_{33}(K)}{2 T_{a} K\left\langle B^{2}\right\rangle}\right]^{-1},
\end{aligned}
$$




$$
N_{a}(K)=\frac{m_{a}}{T_{a}} \nu_{D}^{a}(K) D_{31}(K)\left[1-\frac{3 m_{a} \nu_{D}^{a}(K) D_{33}(K)}{2 T_{a} K\left\langle B^{2}\right\rangle}\right]^{-1} .
$$

The dependence of the bootstrap current on the magnetic configuration can be mainly determined by the viscosity coefficients $M_{a}(K)$ and $N_{a}(K)$. The neoclassical viscosity matrices $M_{a j}$ and $N_{a j}$ are given in the form of energy integrals as

$$
\begin{aligned}
{\left[M_{a j}, N_{a j}\right]=} & n_{a} \frac{2}{\sqrt{\pi}} \int_{0}^{\infty} d K \sqrt{K} e^{-K}\left(K-\frac{5}{2}\right)^{j-1} \\
& \times\left[M_{a}(K), N_{a}(K)\right] .
\end{aligned}
$$

The extension of the $\delta f$ Monte Carlo method to the calculation of $D_{33}$ enables us to evaluate $M_{a}(K)$ from Eq. (37). If we combine that with the results of $D_{31}$, we can also determine the other coefficient $N_{a}(K)$ using Eq. (38). The $\delta f$ Monte Carlo method does not rely on the asymptotic expansion of the drift-kinetic equation. The viscosity-flow relations can be determined for arbitrary collisionality similar to the DKES code. Because the neoclassical viscosity is dominated by the test-particle portion of the collision operator, ${ }^{16}$ the use of the pitch-angle scattering operator without the momentum conservation is justified to calculate the neoclassical viscosity. ${ }^{12}$ Because of the conservation properties of the friction-flow relations in Eq. (34), the bootstrap current $\left\langle J_{\|} B\right\rangle$ can be calculated appropriately with the moment equations. An algebraic procedure to solve the moment equations is given in Ref. 12.

\section{NUMERICAL TESTS}

In this section, we verify the numerical calculation of $D_{33}$ and of the viscosity coefficients $M$ and $N$ using the $\delta f$ Monte Carlo method. As already mentioned, these calculations account for the main portion of the present moment approach. As a $\delta f$ Monte Carlo code, we here used the VENUS $+\delta f$ code, ${ }^{9}$ which has been used ${ }^{7,9,26}$ to calculate the bootstrap current coefficient $D_{31}$ for several nonaxisymmetric devices. We have implemented the $\delta f$ weighting scheme described in Sec. II B into this code for calculating $D_{33}$.

We carried out calculation in a magnetic field model of LHD, where the poloidal and toroidal-field periods are $l=2$ and $m=10$. The field strength in Boozer coordinates is given by

$$
B=B_{0}\left[1-\epsilon_{t}(r) \cos \theta-\epsilon_{h}(r) \cos (l \theta-m \zeta)\right],
$$

where the average minor radius $r$ is chosen as a flux surface label. The toroidal geometry is specified by those values of major radius $R=4 \mathrm{~m}$, the minor radius of last closed flux surface $a=0.8 \mathrm{~m}$, and $B_{0}=1 \mathrm{~T}$. The rotational transform is assumed to be radially constant as $\iota=0.375$. The effect of the net toroidal current on the equilibrium is neglected. We set the radial profile of magnetic field ripple by $\epsilon_{t}=\epsilon_{t a}(r / a)$ and $\epsilon_{h}=\epsilon_{h a}(r / a)^{2}$, where $\epsilon_{t a}$ and $\epsilon_{h a}$ are the values at the last closed flux surface. In the following calculations, the test particles were started from the initial surface: $r / a=0.5$

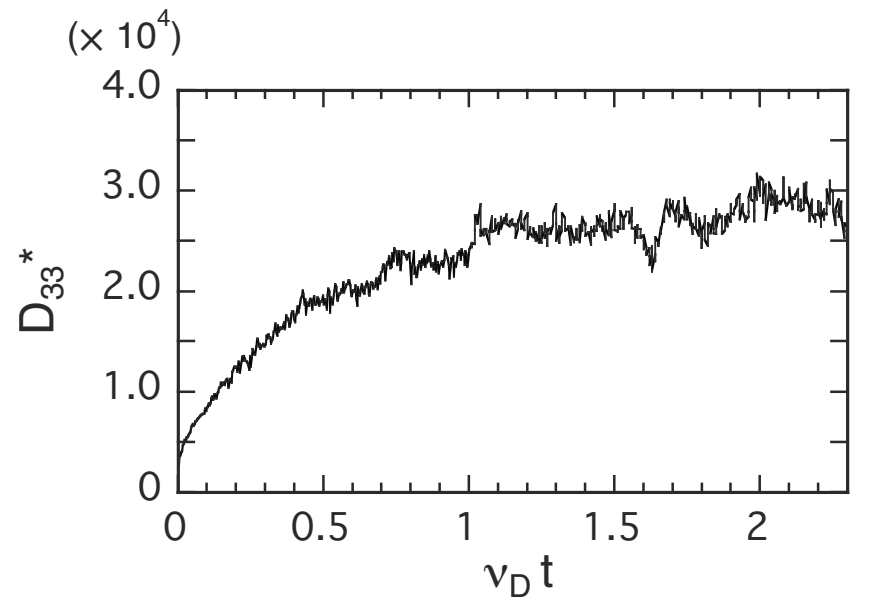

FIG. 1. A typical time history of the normalized coefficient $D_{33}^{*} \equiv D_{33}\left(\frac{1}{2} v_{T} K^{1 / 2}\right)$ calculated with the VENUS $+\delta f$ code for $\epsilon_{t}=0.1$ and $\epsilon_{h}=0.05$. The collisionality is in the banana regime $\left(\nu_{D} / v=1 \times 10^{-5}\right)$.

at time $t=0$. Thus we denote $\epsilon_{t}(r)$ and $\epsilon_{h}(r)$ by values on $r / a=0.5$.

\section{A. The $\delta f$ Monte Carlo code}

The numerical procedure of the VENUS $+\delta f$ code is briefly summarized as follows. A three-dimensional magnetohydrodynamic (MHD) equilibrium described in Boozer coordinates is given as input data. The guiding-center trajectories of test particles are obtained from the drift orbit equations, which are solved with the Runge-Kutta schemes of second and fourth orders with fixed time steps. ${ }^{27}$ At each time step, the pitch-angle scattering is simulated by the Monte Carlo collision operator, ${ }^{28}$ and the perturbed distribution function $\delta f$ is updated by the radial excursions of test particles. In the original code, the monoenergetic bootstrap current, which is here denoted by $\hat{j}_{\mathrm{bs}}$, is calculated by the standard one-weight $\delta f$ scheme with $A_{E} \equiv 0$.

To extend the $\delta f$ weighting scheme used in the VENUS $+\delta f$ code, we have implemented the marker weight $w_{3} \equiv g_{3} / F_{m}$ with the weight equation of Eq. (22) into the code, which allows us to evaluate $D_{33}$ by the Monte Carlo integral of Eq. (25). The weight equation [Eq. (22)] for $D_{33}$ is explicitly written in Boozer coordinates as

$$
\frac{D w_{3}}{D t}=-\frac{v^{2}}{\nu_{D}} P_{2}(\xi)\left(\frac{\psi^{\prime}}{\mathcal{J}_{B}} \frac{\partial}{\partial \zeta}+\frac{\chi^{\prime}}{\mathcal{J}_{B}} \frac{\partial}{\partial \theta}\right) \ln B,
$$

where the prime denotes derivative with respect to the flux surface label, $\psi$ is the toroidal flux, and $\chi$ is the poloidal flux. Figure 1 shows the typical time evolution of $D_{33}$. The simulation reached the steady state in several collision times, and a value of $D_{33}$ was calculated from the time averaging over a finite interval of this steady state. To check the convergence, the Monte Carlo noise $\Delta D_{33}$ is measured with respect to this time averaging. We should note that Eq. (23) does not include the nonlinear drift-term $\mathbf{v}_{d} \cdot \nabla$, which implies that the Lagrangian derivative $D / D t$ represents the derivative along the equilibrium trajectories. ${ }^{11} \mathrm{~A}$ simple way to realize such a test-particle trajectory in the simulation is to choose particle velocities small enough such that $\rho_{p} / a$ remains much smaller 


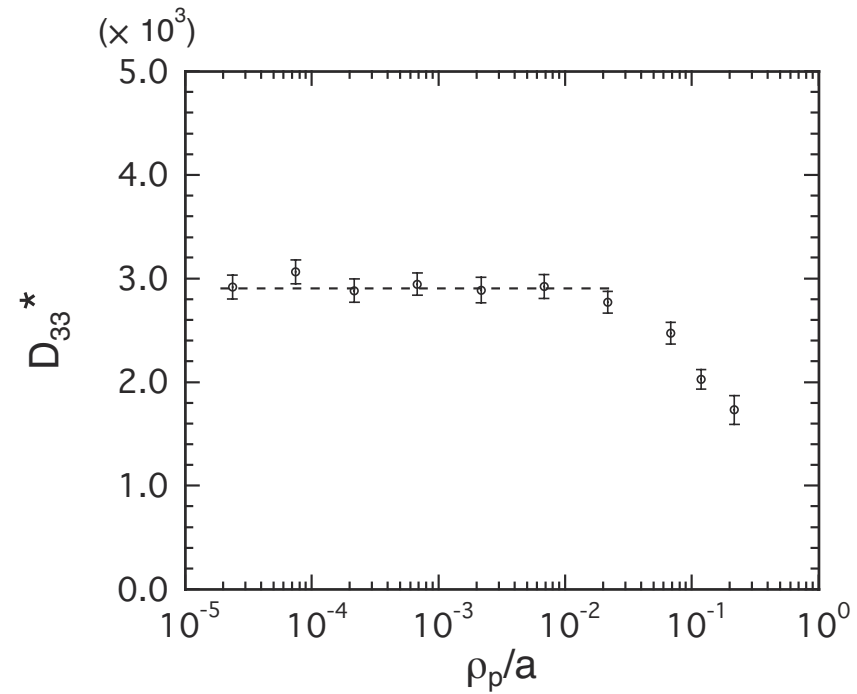

FIG. 2. The normalized coefficient $D_{33}^{*}=D_{33} /\left(\frac{1}{2} v_{T} K^{1 / 2}\right)$ vs $\rho_{p} / a$ calculated with the VENUS $+\delta f$ code for $\epsilon_{t}=0.1$ and $\epsilon_{h}=0.05$. The collisionality is in the banana regime $\left(\nu_{D} / v=1 \times 10^{-4}\right)$.

than unity in all collisionalities. Under this condition, the transport coefficients become insensitive to this parameter. Figure 2 shows the $\rho_{p} / a$ dependence of transport coefficients $D_{33}$ obtained by the code. In Fig. 2 and in the following, the diagonal elements $D_{33}$ are normalized as $D_{33}^{*} \equiv D_{33} /\left(\frac{1}{2} v_{T} K^{1 / 2}\right)$. For small $\rho_{p} / a\left(<10^{-2}\right), D_{33}^{*}$ is not sensitive to this dimensionless parameter and for large $\rho_{p} / a$, the $D_{33}^{*}$ decreases with $\rho_{p} / a$. In the latter case, test particles deviate from the initial surface owing to the radial guidingcenter drifts. In the following calculations, we chose $\rho_{p} / a \simeq 4 \times 10^{-4}$ to ensure negligible finite-orbit width effects.

In the simulation, we used $N \gtrsim 10^{4}$ particles. As already mentioned, an important feature of the moment approach is to calculate the neoclassical viscosity for arbitrary collisionality. To keep this feature, we require the accurate calculation of $D_{33}$ for a wide range of collision frequencies. To assist this, we used a supplementary method to improve the numerical convergence of $D_{33}$ in the collisional regime: typically for $\nu_{D} / v \gtrsim 10^{-2}$. This method will be discussed in the Appendix, and it was utilized to obtain the numerical results in this paper.

\section{B. Numerical results}

We illustrate the numerical results for a model field defined in the beginning of this section. For comparison, we carried out calculations for the same parameters with those used in Ref. 12 and compared the numerical results obtained by the VENUS $+\delta f$ code with those obtained by the DKES code, where the results of the latter are given in Ref. 12. Figure 3 shows the collisionality dependence of the calculated $D_{33}^{*}$ in the axisymmetric limit, $\epsilon_{h}=0$. The solid line shows the asymptotic formula ${ }^{13}$ in the banana regime for $D_{33}^{*}$,

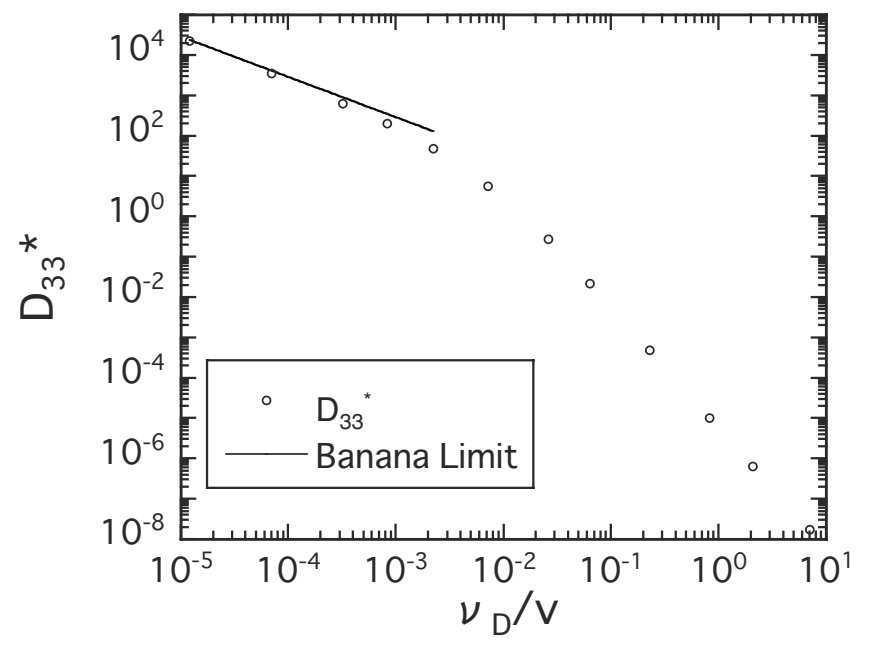

FIG. 3. The normalized coefficient $D_{33}^{*} \equiv D_{33} /\left(\frac{1}{2} v_{T} K^{1 / 2}\right)$ vs $\nu_{D} / v$ calculated with the VENUS $+\delta f$ code for $\epsilon_{t}=0.1$ and $\epsilon_{h}=0$. The solid line shows the asymptotic values in the banana regime.

$$
D_{33}^{*}=f_{t}\left\langle B^{2}\right\rangle \frac{2}{3\left(\nu_{D} / v\right)} .
$$

We thus confirmed the good convergence of $D_{33}^{*}$ in this regime. Figure 4 shows the results for several values of $\epsilon_{h}$ such that $\epsilon_{h}=0,0.01,0.05$, and 0.1 , where $\epsilon_{t}$ in Eq. (40) is kept constant as $\epsilon_{t}=0.1$. The difference due to the amplitude of helical ripple $\epsilon_{h}$ manifests itself mainly in the plateau and the collisional regimes. The results in Fig. 4 show good agreement with those in Ref. 12.

Using the results of Fig. 4, we calculate the energydependent viscosity coefficients $M(K)$ and $N(K)$. First, Fig. 5 shows the $\epsilon_{h}$ dependence of a normalized viscosity coefficient $M^{*}$, where $M^{*} \equiv M(K) /\left(m v_{T} K^{3 / 2}\right)$. The viscosity coefficient $M(K)$ measures the damping rate of neoclassical flows along the field line. The normalized coefficient $M^{*}$ is written in terms of the normalized coefficient $D_{33}^{*}$ as

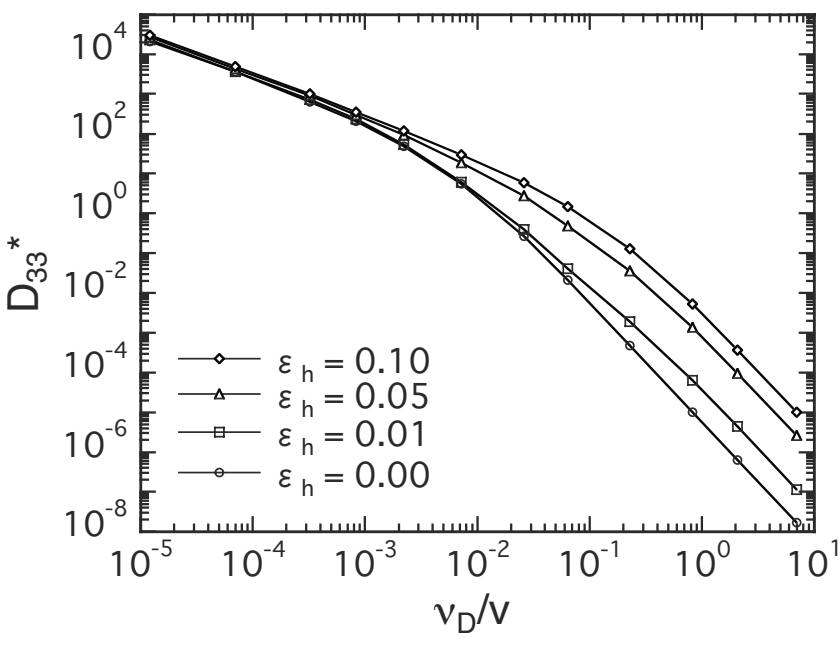

FIG. 4. The normalized coefficient $D_{33}^{*} \equiv D_{33}\left(\frac{1}{2} v_{T} K^{1 / 2}\right)$ vs $\nu_{D} / v$ calculated with the VENUS $+\delta f$ code for $\epsilon_{h}=0,0.01,0.05$, and 0.1 with $\epsilon_{t}=0.1$. 


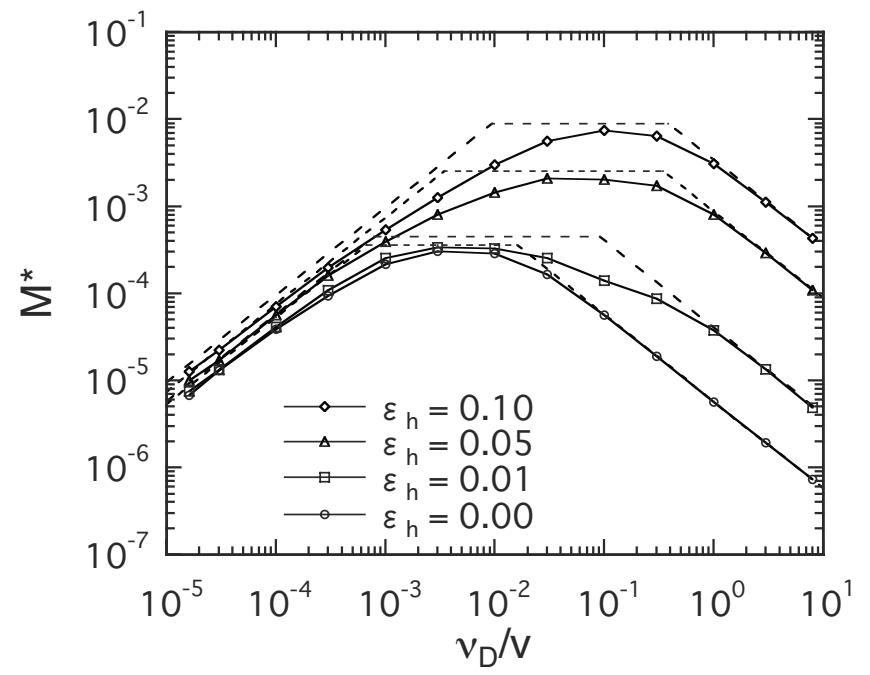

FIG. 5. The normalized viscosity coefficient $M^{*} \equiv M(K) /\left(m v_{T} K^{3 / 2}\right)$ vs $\nu_{D} / v$ calculated with the VENUS $+\delta f$ code. The dashed segments show the asymptotic values of $M^{*}$ for the three collisionality regimes.

$$
M^{*}=\frac{\left(\nu_{D} / v\right)^{2} D_{33}^{*}}{1-\frac{3}{2}\left(\nu_{D} / v\right) D_{33}^{*} /\left\langle B^{2}\right\rangle} .
$$

The dashed segments in Fig. 5 show the asymptotic values in the Pfirsch-Schlüter, plateau, and banana regimes. The asymptotic formulae used here are given by ${ }^{12}$

$$
\begin{aligned}
M_{\mathrm{P} . \mathrm{S} .}^{*}= & \frac{2}{5}\left\langle(\mathbf{B} \cdot \nabla \ln B)^{2}\right\rangle\left[\nu_{T}(K) / v\right]^{-1}, \\
M_{\text {plateau }}^{*}= & \frac{\pi}{4}\left\langle B^{2}\right\rangle^{1 / 2}\left(4 \pi^{2} / V^{\prime}\right) \\
& \times\left(\sum_{(m, n) \neq(0,0)}\left|\beta_{m n}\right|^{2}\left|m \chi^{\prime}-n \psi^{\prime}\right|\right), \\
M_{\text {banana }}^{*}= & \frac{2}{3}\left(f_{t} / f_{c}\right)\left\langle B^{2}\right\rangle\left[\nu_{D}(K) / v\right],
\end{aligned}
$$

where $V$ is the volume enclosed by the flux surface and $\beta_{m n}$ is the magnetic-field spectrum in Hamada coordinates. ${ }^{29}$ The ratio of the fraction of trapped particles to that of circulating ones is denoted by $f_{t} / f_{c}$. To be consistent with Ref. 12, the test-particle frequency $\nu_{T}$ in Eq. (44) is set as $\nu_{T}=3 \nu_{D}$. In Fig. 5, the results obtained from the $\delta f$ Monte Carlo method are in reasonable agreement with the asymptotic formulae. Next, we calculate the $\epsilon_{h}$ dependence of a normalized viscosity coefficient $N(K)^{*}$, where $N^{*} \equiv N(K) /$ $\left[\left(B v_{T} / \Omega\right) K^{3 / 2}\right]$. The viscosity coefficient $N(K)$ measures the driving force of parallel flows mainly due to the trapped particles. The normalized coefficient $N^{*}$ is written in terms of $D_{31}^{*}$ and $D_{33}^{*}$ as

$$
N^{*}=\frac{\left(\nu_{D} / v\right) D_{31}^{*}}{1-\frac{3}{2}\left(\nu_{D} / v\right) D_{33}^{*} /\left\langle B^{2}\right\rangle},
$$

where the bootstrap current coefficient $D_{31}$ is normalized as $D_{31}^{*} \equiv D_{31} /\left[\frac{1}{2} v_{T}\left(B v_{T} / \Omega\right) K\right]$. Figure 6 shows the results for $N^{*}$. Also plotted for benchmarking are the results ${ }^{12}$ obtained from the DKES code. The $\delta f$ Monte Carlo method has reproduced the curve obtained by the DKES code. Consequently, the numerical results for $M(K)$ and $N(K)$ obtained

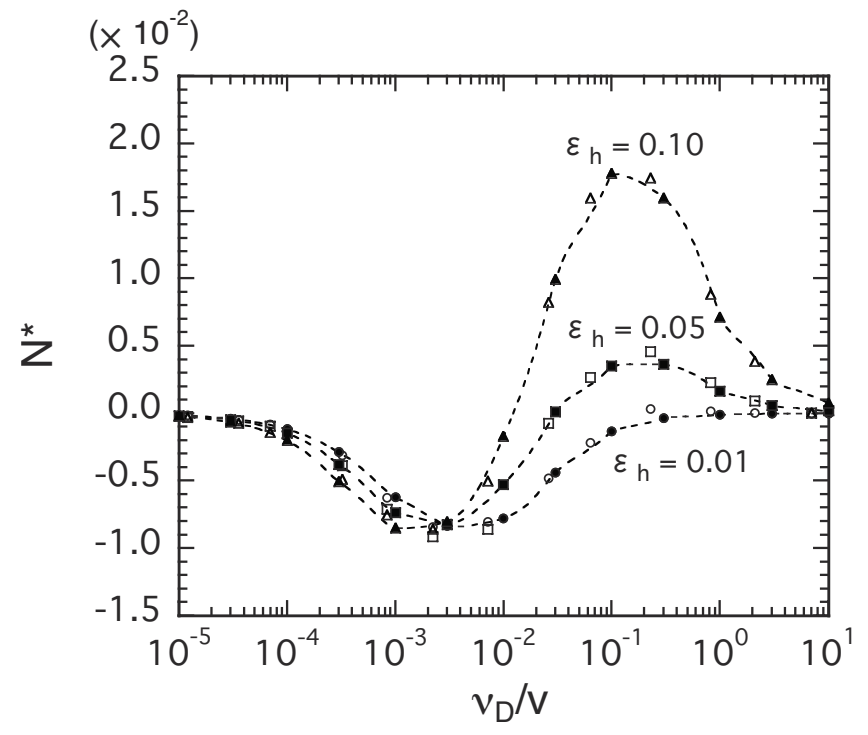

FIG. 6. The normalized viscosity coefficient $N^{*} \equiv N(K) /\left[\left(B v_{T} / \Omega\right) K^{3 / 2}\right]$ vs $\nu_{D} / v$. The open symbols show the numerical results from the VENUS $+\delta f$ and the closed symbols show those from the DKES code in Ref. 12.

here are in reasonable agreement with the asymptotic formulae and with those obtained by the DKES code. We thus conclude that these numerical tests provide a sufficient basis for applying the $\delta f$ Monte Carlo method to the moment approach.

\section{CONCLUSION}

A $\delta f$ Monte Carlo method has been presented to evaluate the bootstrap current in nonaxisymmetric toroidal plasmas with the moment approach. Using the $\delta f$ weighting scheme proposed in this work, the diagonal $\left(D_{33}\right)$ and the nondiagonal $\left(D_{31}\right)$ elements of the monoenergetic transport matrix are calculated. The former have been calculated so far only by the variational principle. In the numerical tests, we have calculated the energy-dependent viscosity coefficients $M$ and $N$ from $D_{31}$ and $D_{33}$ and have obtained the reasonable agreement between the variational principle and the asymptotic formulae. With the moment approach, the bootstrap current $\left\langle J_{\|} B\right\rangle$ is evaluated from these viscosity coefficients with the algebraic moment equations that satisfy the physical requirement of the conservation laws.

We have implemented the above $\delta f$ weighting scheme into one of $\delta f$ Monte Carlo codes, namely, the VENUS $+\delta f$. This code has been applied to the calculation of $D_{31}$ in several nonaxisymmetric devices such as LHD. ${ }^{26}$ In the LHD experiment, the time evolution of the bootstrap current profiles has recently become possible to be measured. ${ }^{30}$ The present work will be useful for experimental analysis of the bootstrap current in LHD as future applications.

\section{ACKNOWLEDGMENTS}

The authors thank S. Nishimura for providing the numerical data in Ref. 12. The computation was performed on the Opteron cluster system at the National Institute of Fusion Science (NIFS), Japan. 
This work was supported by NIFS/NINS under the NIFS Collaborative Research Program (Grant No. NIFS07KUHL011) and under the Project of Formation of International Network of Scientific Collaboration, Japan. This work was also supported in part by the Swiss National Science Foundation.

\section{APPENDIX: CALCULATION OF $D_{33}$ IN COLLISIONAL REGIME}

In this appendix, we discuss an additional topic of the $\delta f$ Monte Carlo method to calculate $D_{33}$. The subject addressed here is about the expression for the calculation of $D_{i j}$ in the variational principle, which is given by Eq. (7). In this equation, the time-reversal flux ${ }^{14} \sigma_{i}^{+}(i=1,3)$ is used not only for the source term of the drift-kinetic equation but for the integrand in the inner-product operation as well. We found that a similar expression for the $\delta f$ Monte Carlo method was useful to improve the numerical convergence of $D_{33}$ in the collisional regime.

To derive an expression of $D_{33}$ like Eq. (7), we use the symmetric properties of the operators $V_{\|}$and $C$ in Eq. (12). In Eq. (18), $D_{33}$ is given by $D_{33}=-\left(B v \xi, g_{3}\right)$. Using the symmetric properties of Eq. (12),

$$
\begin{aligned}
D_{33} & =-\left(B v \xi, g_{3}\right)=\left(C\left(B v \xi / \nu_{D}\right), g_{3}\right)=\left(B v \xi / \nu_{D}, C g_{3}\right) \\
& =\left(B v \xi / \nu_{D}, V_{\|} g_{3}\right)+\left(B v \xi / \nu_{D}, \sigma_{3}^{+}\right)=-\left(\sigma_{3}^{+}, g_{3}\right),
\end{aligned}
$$

where $\left(B v \xi / \nu_{D}, \sigma_{3}^{+}\right)$automatically vanishes. Note that $\xi$ is the eigenfunction of the pitch-angle scattering operator $\nu_{D}^{-1} C$ with the eigenvalue of -1 . Therefore, $C\left(B v \xi / \nu_{D}\right)=-B v \xi$. From Eq. (A1), we have obtained another expression for $D_{33}$,

$$
D_{33}=-\left(\sigma_{3}^{+}, g_{3}(\mathbf{x}, \xi)\right)=-\frac{\sum_{n=1}^{N} \mathcal{J}_{B}\left(\mathbf{x}_{n}\right) \sigma_{3}^{+}\left(\mathbf{x}_{n}, \xi_{n}\right) w_{3}^{(n)}}{\sum_{n=1}^{N} \mathcal{J}_{B}\left(\mathbf{x}_{n}\right)} .
$$

The physical meaning of the equivalence between Eqs. (25) and (A2) can be seen by taking the inner product of Eq. (19) with $B v \xi$,

$$
\left(B v \xi, V_{\|} g_{3}\right)-\left(B v \xi, C g_{3}\right)=0 .
$$

From Eqs. (A1) and (A3), we see that $\left(B v \xi, g_{3}\right)$ is proportional to the momentum lost by collisions; $\left(\sigma_{3}^{+}, g_{3}\right)$ is proportional to the momentum generated indirectly by the trapped particles. From these physical pictures, we understand that the numerical values $\left(B v \xi, g_{3}\right)$ and $\left(\sigma_{3}^{+}, g_{3}\right)$ measure the quantities that are in balance with each other. In actual simulations, however, such an exact balance cannot be achieved because the terms $\left(\sigma_{3}^{+}, g_{3}\right)$ and $\left(B v \xi, g_{3}\right)$ are characterized by the different relaxation times in transient phase of a initialvalue problem. To obtain the results in Sec. III B, we attempted the calculation of $D_{33}$ using both terms and consequently found that the calculated transport coefficients experience different levels of the Monte Carlo noise. Figure 7 shows the collisionality dependence of the noise observed for $D_{33}$ in the nonaxisymmetric case with $\epsilon_{h}=0.05$. In the low collisionality regime of $\nu_{D} \lesssim 10^{-2}$, the noise for $D_{33} \equiv-\left(\sigma_{3}^{+}, g_{3}\right)$ is considerably larger than that of $D_{33}=-\left(B v \xi, g_{3}\right)$. In contrast, we obtained good convergence

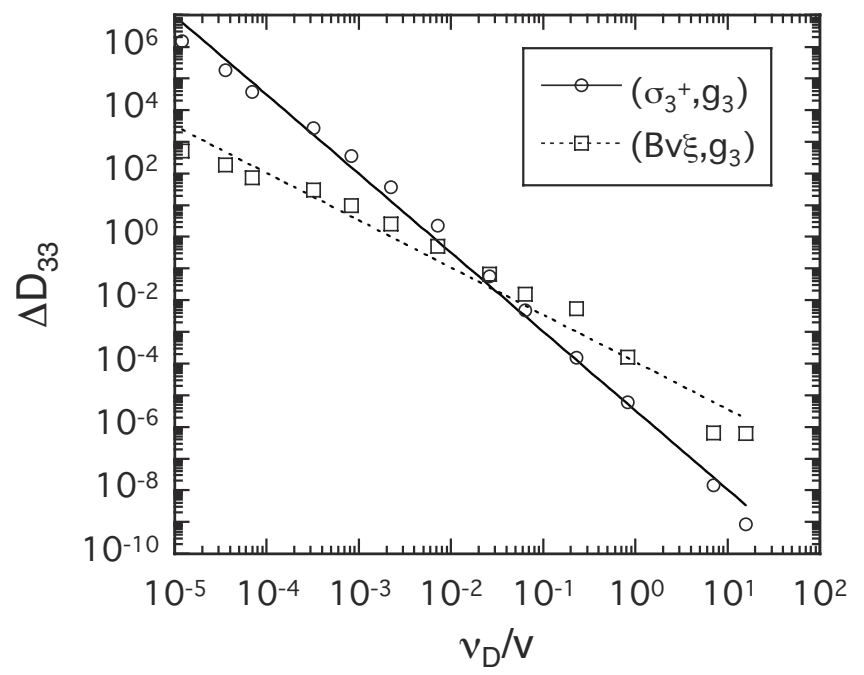

FIG. 7. The absolute values of the Monte Carlo noise denoted by $\Delta D_{33}$ vs $\nu_{D} / v$ for two different expressions for $D_{33}$ in Eqs. (25) and (A2). Here, $\epsilon_{t}=0.1$ and $\epsilon_{h}=0.05$. The number of test particles is $N \gtrsim 10^{4}$ for all points. (The same number of test particles was used for each $\nu_{D} / v$.)

of $D_{33}$ by the expression $D_{33}=-\left(\sigma_{3}^{+}, g_{3}\right)$ for the collisional regime with $\nu_{D} \gtrsim 10^{-2}$. This result suggests that the use of Eq. (A2) is favorable in the collisional regime to improve the statistical convergence of $D_{33}$. This result can be interpreted by the difference of relaxation times between $\left(\sigma_{3}^{+}, g_{3}\right)$ and $\left(B v \xi, g_{3}\right)$.

Finally, we note that the bootstrap current $D_{31}$ can also be calculated by $D_{31}=-\left(\sigma_{3}^{+}, g_{1}\right)$ in a similar way to Eq. (A2). Moreover, we see from the Onsager relation that the Ware flux coefficient $D_{13}$ can also be used as a measure of the nondiagonal elements of transport matrix; the Ware flux coefficient $D_{13}$ can be calculated using the marker weight $w_{3}$ introduced in this paper. Such arbitrariness of the expression to calculate the monoenergetic transport coefficients is a freedom that may be used to improve statistics in the $\delta f$ Monte Carlo methods.

${ }^{1}$ K. C. Shaing, E. C. Crume, Jr., J. S. Tolliver, S. P. Hirshman, and W. I. van Rij, Phys. Fluids B 1, 148 (1989).

${ }^{2}$ K. C. Shaing, B. A. Carreras, N. Dominguez, V. E. Lynch, and J. S. Tolliver, Phys. Fluids B 1, 1663 (1989).

${ }^{3}$ K. Y. Watanabe, N. Nakajima, M. Okamoto, K. Yamazaki, Y. Nakamura, and M. Wakatani, Nucl. Fusion 35, 335 (1995).

${ }^{4}$ W. A. Cooper, S. F. Margalet, S. J. Allfrey, M. Yu. Isaev, M. I. Mikhailov, V. D. Shafranov, A. A. Subbotin, Y. Narushima, S. Okamura, C. Suzuki, K. Yamazaki, G. Y. Fu, L. P. Ku, D. A. Monticello, M. H. Redi, A. H. Reiman, M. C. Zarnstorff, J. Nührenberg, and T. N. Todd, Plasma Phys. Controlled Fusion 44, B357 (2002).

${ }^{5}$ A. H. Boozer and H. J. Gardner, Phys. Fluids B 2, 2408 (1990).

${ }^{6}$ Y. Wu and R. B. White, Phys. Fluids B 5, 3291 (1993).

${ }^{7}$ K. Allmaier, C. D. Beidler, M. Yu. Isaev, S. V. Kasilov, W. Kernbichler, H. Maassberg, S. Murakami, D. A. Spong, and V. Tribaldos, in 16th International Stellarator/Heliotron Workshop, Toki, 2007, edited by S. Morita (National Institute for Fusion Science, Toki, 2008), pp. P2-029.

${ }^{8}$ K. C. Shaing and J. D. Callen, Phys. Fluids 26, 3315 (1983).

${ }^{9}$ M. Yu. Isaev, S. Brunner, W. A. Cooper, T. M. Tran, A. Bergmann, C. D. Beidler, J. Geiger, H. Maassberg, J. Nührenberg, and M. Schmidt, Fusion Sci. Technol. 50, 440 (2006).

${ }^{10}$ K. Allmaier, S. V. Kasilov, W. Kernbichler, and G. O. Leitold, Phys. Plasmas 15, 072512 (2008).

${ }^{11}$ Z. Lin, W. M. Tang, and W. W. Lee, Phys. Plasmas 2, 2975 (1995).

${ }^{12}$ H. Sugama and S. Nishimura, Phys. Plasmas 9, 4637 (2002). 
${ }^{13}$ H. Sugama and S. Nishimura, Phys. Plasmas 15, 042502 (2008).

${ }^{14}$ S. P. Hirshman, K. C. Shaing, W. I. van Rij, C. O. Beasely, Jr., and E. C. Crume, Jr., Phys. Fluids 29, 2951 (1986).

${ }^{15}$ W. I. van Rij and S. P. Hirshman, Phys. Fluids B 1, 563 (1989).

${ }^{16}$ S. P. Hirshman and D. J. Sigmar, Nucl. Fusion 21, 1079 (1981).

${ }^{17}$ M. Taguchi, Phys. Fluids B 4, 3638 (1992).

${ }^{18}$ O. Motojima, H. Yamada, A. Komori, N. Ohyabu, K. Kawahata, O. Kaneko, S. Masuzaki, A. Ejiri, M. Emoto, H. Funaba, M. Goto, K. Ida, H. Idei, S. Inagaki, N. Inoue, S. Kado, S. Kubo, R. Kumazawa, T. Minami, J. Miyazawa, T. Morisaki, S. Morita, S. Murakami, S. Muto, T. Mutoh, Y. Nagayama, Y. Nakamura, H. Nakanishi, K. Narihara, K. Nishimura, N. Noda, T. Kobuchi, S. Ohdachi, Y. Oka, M. Osakabe, T. Ozaki, B. J. Peterson, A. Sagara, S. Sakakibara, R. Sakamoto, H. Sasao, M. Sasao, K. Sato, M. Sato, T. Seki, T. Shimozuma, M. Shoji, H. Suzuki, Y. Takeiri, K. Tanaka, K. Toi, T. Tokuzawa, K. Tsumori, K. Tsuzuki, I. Yamada, S. Yamaguchi, M. Yokoyama, K. Y. Watanabe, T. Watari, Y. Hamada, K. Matsuoka, K. Murai, K. Ohkubo, I. Ohtake, M. Okamoto, S. Satoh, T. Satow, S. Sudo, S. Tanahashi, K. Yamazaki, M. Fujiwara, and A. Iiyoshi, Phys. Plasmas 6, 1843 (1999).

${ }^{19}$ A. H. Boozer, Phys. Fluids 26, 1288 (1983).
${ }^{20}$ A. Y. Aydemir, Phys. Plasmas 1, 822 (1994).

${ }^{21}$ M. Tessarotto, R. B. White, and L. J. Zheng, Phys. Plasmas 1, 2603 (1994).

${ }^{22}$ M. Sasinowski and A. H. Boozer, Phys. Plasmas 2, 610 (1995).

${ }^{23}$ K. Hanatani, J. Plasma Fusion Res. Series 1, 472 (1998).

${ }^{24}$ S. Nishimura, H. Sugama, and CHS Group, Fusion Sci. Technol. 46, 77 (2004).

${ }^{25}$ N. Nakajima and M. Okamoto, J. Phys. Soc. Jpn. 61, 833 (1992).

${ }^{26}$ M. Yu. Isaev, K. Y. Watanabe, M. Yokoyama, N. Ohyabu, C. D. Beidler, H. Maassberg, W. A. Cooper, T. M. Tran, and M. I. Mikhailov, Plasma Fusion Res. 3, 036 (2008).

${ }^{27}$ O. Fischer, W. A. Cooper, M. Yu. Isaev, and L. Villard, Nucl. Fusion 42, 817 (2002).

${ }^{28}$ A. H. Boozer and G. Kuo-Petravic, Phys. Fluids 24, 851 (1981).

${ }^{29}$ S. Hamada, Nucl. Fusion 2, 23 (1962).

${ }^{30}$ Y. Nakamura, K. Y. Watanabe, K. Kawaoto, K. Ida, Y. Narushima, M. Yoshinuma, S. Sakakibara, I. Yamada, T. Tokuzawa, M. Goto, K. Tanaka, N. Nakajima, K. Kawahata, and LHD Experimental Group, in Proceedings of the 22nd Fusion Energy Conference (IAEA, Geneva, Switzerland, 2008), pp. EX/P6-20. 